\title{
Neue Ansätze in der Maschinenkonstruktionslehre
}

\section{o.Prof.Dr.-Ing.A.Albers; Prof.Dr.-Ing.H.Birkhofer; Dipl.-Ing.S.Matthiesen}

Die Maschinenelemente selbst und das industrielle Umfeld, in denen sie entwickelt, produziert und angewandt werden, haben sich in den letzten Jahren drastisch gewandelt. Zum einen steigt die Vielzahl der Maschinenelemente durch neue Werkstoffe und neue Fertigungstechnologien stark an, zum anderen entstehen durch das veränderte industrielle Umfeld mit dem Trend zum Einsatz vorgefertigter Teilsysteme neue Prozesse in der Konstruktion. Im Zuge der Outsourcing-Strategien von Unternehmen werden Maschinenelemente zu einem großen Anteil als Zulieferkomponenten von hochspezialisierten Zulieferern bezogen und in die eigene Entwicklung neuer Produkte integriert. Neben dem Gestalten von Maschinenelementen in der Zulieferindustrie hat dadurch das Gestalten mit Maschinenelementen bei den Anwendern eine vergleichbare Bedeutung erlangt $/ 5 /$.

Ergänzend zum Fachwissen über Bauteile und ihre Eigenschaften, müssen Ingenieure damit in hohem Maße Systeme mit ihren eigenständigen Anforderungen kennen und hinsichtlich ihrer Anwendung beurteilen können.

Zusätzlich zu technischen Dimensionierungs- und Gestaltungskriterien haben unter den heutigen globalen Wettbewerbsbedingungen wirtschaftliche Kriterien eine entscheidende Bedeutung erlangt. Ökologische Kriterien werden zunehmend wichtiger. Die Managementstrukturen in der Industrie verändern sich vollständig. Simultaneous-Engineering mit parallel ablaufenden Entwicklungsprozessen für Produkt und Prozeß in interdisziplinären Teams, erfordert neben Fachwissen zusätzliche Qualifikationen des Entwicklungsingenieurs, wie Teamfähigkeit und interdisziplinäre Kommunikationskompetenz. Künftige Produktentwickler müssen darauf in angemessener Weise reagieren und eine ganzheitliche Beurteilung von Lösungsvarianten anstreben.

Die universitäre Ausbildung darf sich also nicht nur auf die Vermittlung von Fachkompetenz beschränken, sondern muß vielmehr auf die weiteren Anforderungen aus der industriellen Arbeitswelt des Ingenieurs eingehen. 
Neben einer klaren Methodenkompetenz zur Beherrschung des Entwicklungsprozesses zählen Sozialkompetenz und Elaborationskompetenz mit den entsprechenden Softskills zu den Kernkompetenzen der Produktentwickler (Abb. 1) und müssen in der universitären Lehre vermittelt werden.

Um den Bedarf an gut ausgebildeten Maschinenbauingenieuren sicherzustellen und die Aktualität der Lehre auf dem Gebiet der Maschinenelemente zu gewährleisten, ist es somit erforderlich, sie über ihre bewährten Inhalte hinaus weiter zu entwickeln und insbesondere den nachfolgenden Anforderungen Rechnung zu tragen.

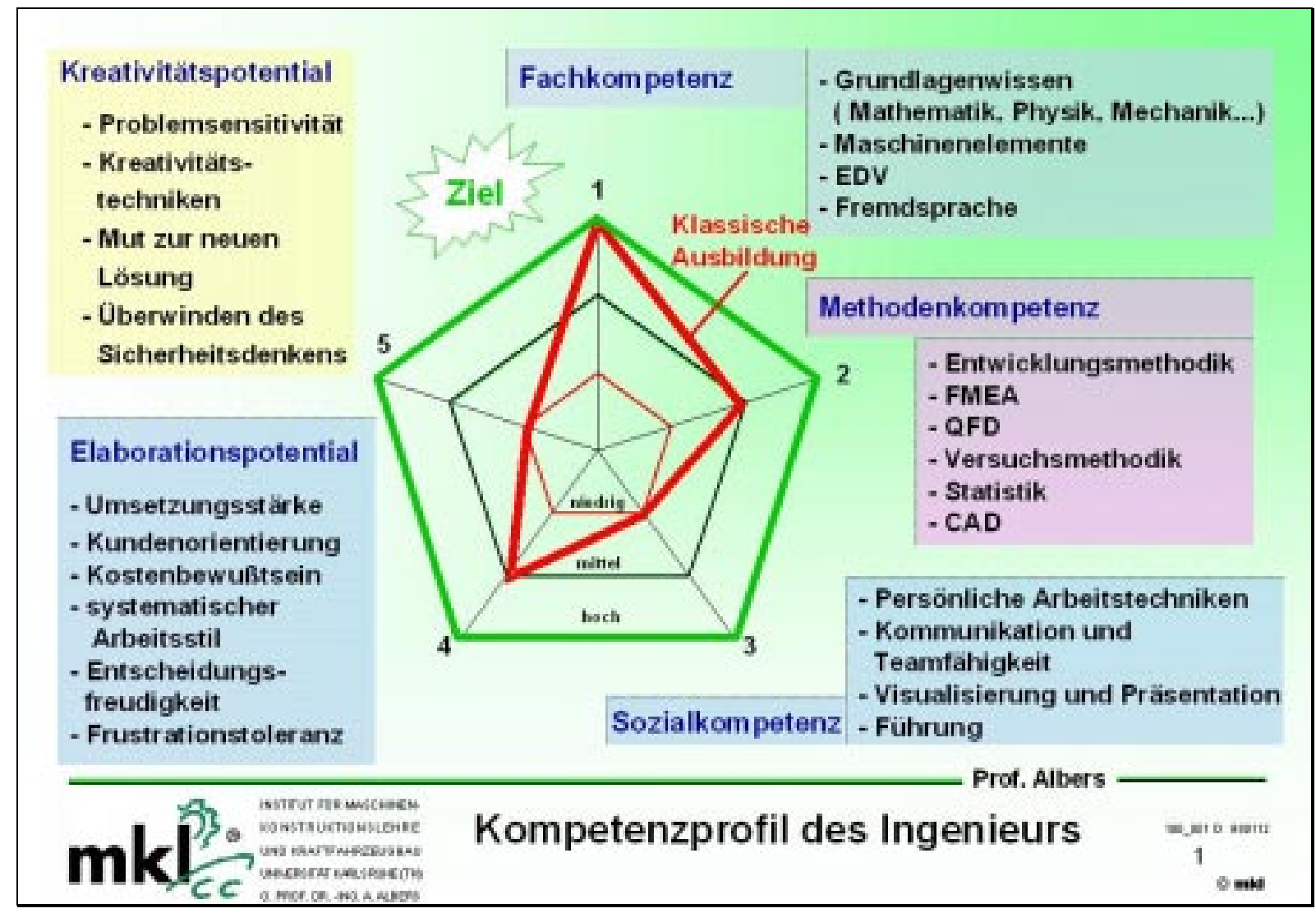

Abb.1: Das veränderte Kompetenzprofil des Ingenieurs /1/

Die Lehre auf dem Gebiet der Maschinenkonstruktion stellt einen zentralen Schwerpunkt in der Grundausbildung von Maschinenbauingenieuren dar. Es steht außer Frage, daß sie nicht allein alle Elemente des Kompetenzprofils eines Ingenieurs vermitteln kann. Dennoch integriert die Maschinenkonstruktionslehre das Fach- und Methodenwissen der theoretischen und angewandten Grundlagenfächer bei der Dimensionierung und Gestaltung von Bauteilen und Komponenten nach vorgegebenen Anforderungen in ein ganzheitliches, ingenieurmäßiges Denken und 
Handeln. Sie hat damit die Chance einen entscheidenden Beitrag zur Vermittlung von Fach-, Methodenkompetenz und Sozialkompetenz zu leisten und zusätzlich erste Ansätze zum Bildung von Kreativitäts- und Elaborationspotential zu liefern.

\section{Der Arbeitskreis Lehre Maschinenelemente (akme)}

Um sich diesen Forderungen zu stellen und in Vorträgen, Diskussionen und Workshops über eine Weiterentwicklung der Lehre auf dem Gebiet der Maschinenelemente zu diskutieren, hat sich, auf Anregung von Herrn Prof. Dr.-Ing. A. Albers und Herrn Prof. Dr.-Ing. H. Birkhofer, eine Gruppe von 20 Hochschullehrern aus den Bereichen Maschinenelemente, Konstruktionsmethodik und Informationstechnologie im Jahre 1997 zum Arbeitskreis Lehre Maschinenelemente (akme) unter dem Dach der WGMK zusammengefunden. Seither wird kontinuierlich an den Zielen einer Neuorientierung und Weiterentwicklung der Maschinenkonstruktionslehre gearbeitet.

Im Arbeitskeis wurden 16 Thesen zur Weiterentwicklung der Maschinenelementelehre formuliert, die folgende Bereiche abdecken:

- Aufgaben und Lernziele

- Einordnung der Maschinenelementelehre im Maschinenbau

- Inhalte der Maschinenelementelehre

- zu vermittelnde Fähigkeiten und Fertigkeiten

- Didaktik

Ziel des Arbeitskreises ist die Entwicklung eines Baukastens für Lehr- und Lernmodelle, der den oben genannten Thesen gerecht wird. Aus diesem Baukasten sollen Lehr- und Lernkonzepte zusammengestellt werden können. Dabei muß die individuelle Ausrichtung der Lehre an den verschiedenen Universitäten beibehalten bleiben.

Komponenten und Umsetzungsvorschläge zu den drei grundlegenden Lehr- und Lernmodellen - konventionelles, systembetontes und projektbetontes Lehr- und Lernmodell - sind im Rahmen der Tätigkeit des Arbeitskreises bereits entstanden. Einige Universitäten konnten die Vorschläge bereits umsetzen und damit wertvolle Erfahrungen in den Arbeitskreis einbringen (/2/, /3/,/4/). 


\section{Das Karlsruher Lehrmodell}

Bereits im Jahre 1995 mit der Berufung von Prof. Albers an den Lehrstuhl für Maschinenkonstruktionslehre an der Universität Karlsruhe wurden auf der Grundlage des im Abb. 1 dargestellten Kompetenzprofils neue Ansätze für die Lehre definiert Zusammen mit den Erfahrungen und Thesen aus dem akme wurden diese Ansätze in einem neuen Lehr- und Lernmodell umgesetzt. Seit April '99 wird dieses Modell eingeführt.

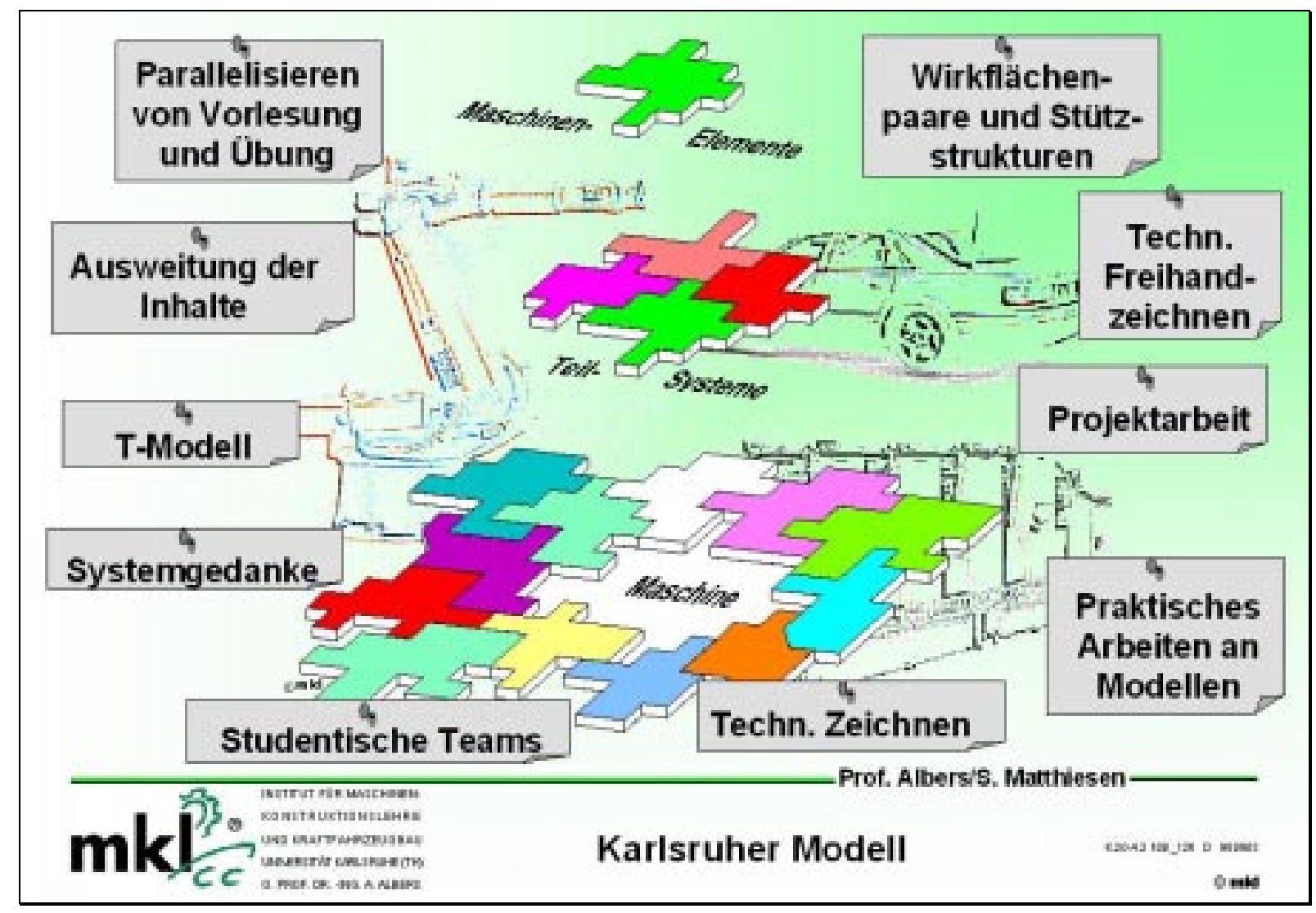

Abb.2: Grundsätzliche Ideen des Karlsruher Lehrmodells

Das Karlsruher Modell zur Vermittlung der Maschinenkonstruktionslehre gliedert sich in drei Veranstaltungsmodule: Vorlesung, Übung, und Workshop. Diese basieren jeweils auf unterschiedlichen Lehr- und Lerninhalten. 


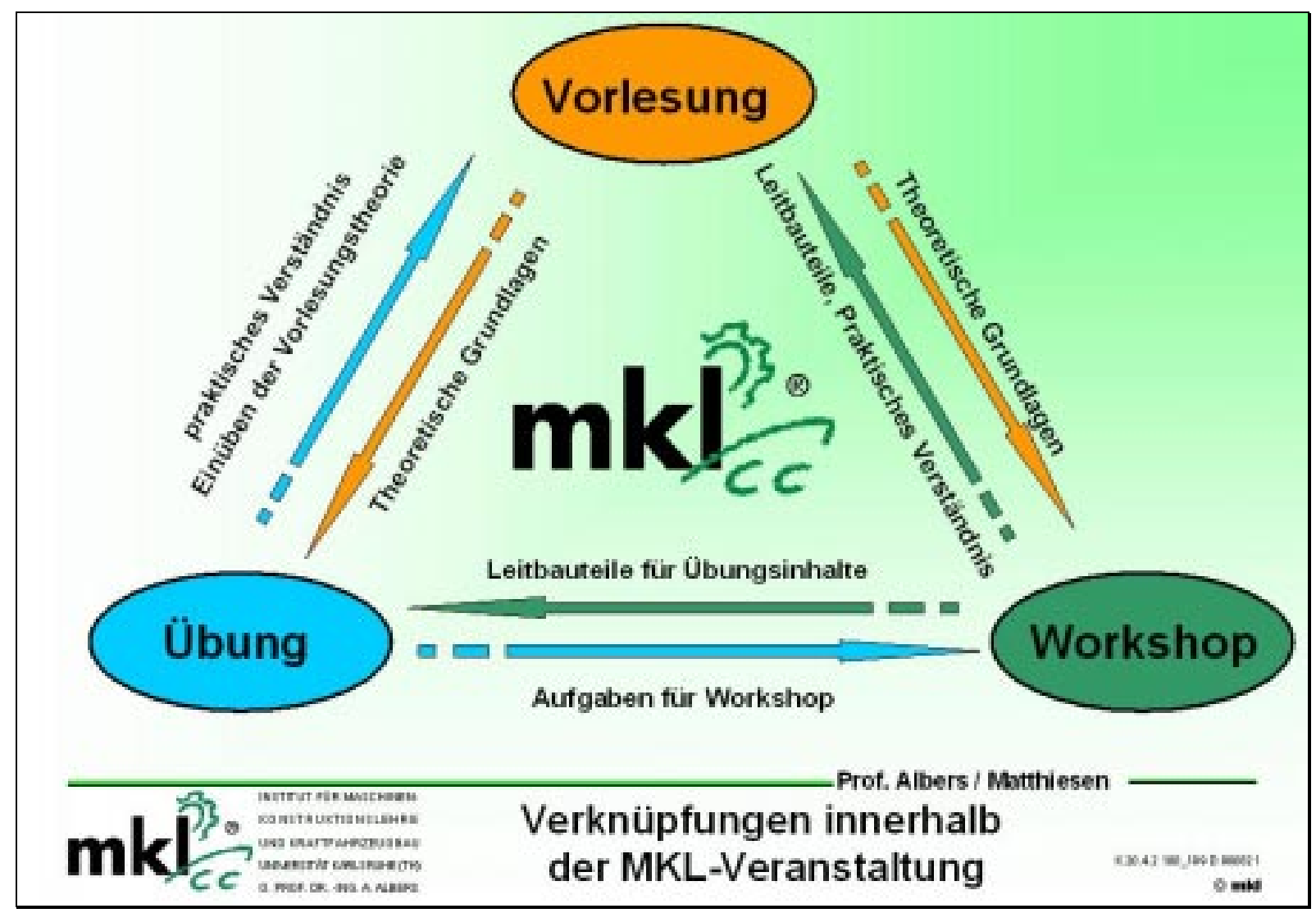

Abb.3: Verknüpfungen des neuen Vorlesungskonzeptes

Die Vorlesung, grundsätzlich als übergeordneter Baustein des Lehrmodells verstanden, liefert die theoretischen Grundlagen für die beiden anderen Veranstaltungsmodule und erläutert an Beispielen aus der industriellen Entwicklungspraxis die Prozesse der Produktentwicklung. Wichtig sind hier zwei Leitbeispiele - ein PKW-Antriebsstrang und ein Extruder - die durch die gesamte Vorlesung die Besprechung der einzelnen Maschinenelemente begleiten.

Die Übung vertieft das theoretische Wissen um gleichzeitig die praktische Anwendung des Vorlesungsstoffes in konkreten Übungsaufgaben zu vermitteln. An die dritte Komponente, den Workshop werden aus der Saalübung Aufgaben weitergegeben.

Im Workshop werden in studentischen Teams konkrete Aufgaben bearbeitet. $\mathrm{Zu}$ Beginn der Veranstaltung werden den Studenten Maschinenelemente vor allem praktisch begreifbar gemacht. Dadurch liefert der Workshop der Vorlesung und der Übung Leitbauteile und Leitsysteme, die den Studierenden in Funktion und Gestalt vollkommen bekannt sind. 


\section{Vorlesung}

Bisherige Vorlesungskonzepte bestanden meist in einer Aneinanderreihung verschiedener Maschinenelemente. Bei jedem Maschinenelement wurde explizit auf eine Beschreibung des Maschinenelementes, auf seine Funktion, seine Gestaltung und seine Auslegung eingegangen. Dabei wurden versucht alle Maschinenelemente vollständig zu bearbeiten.

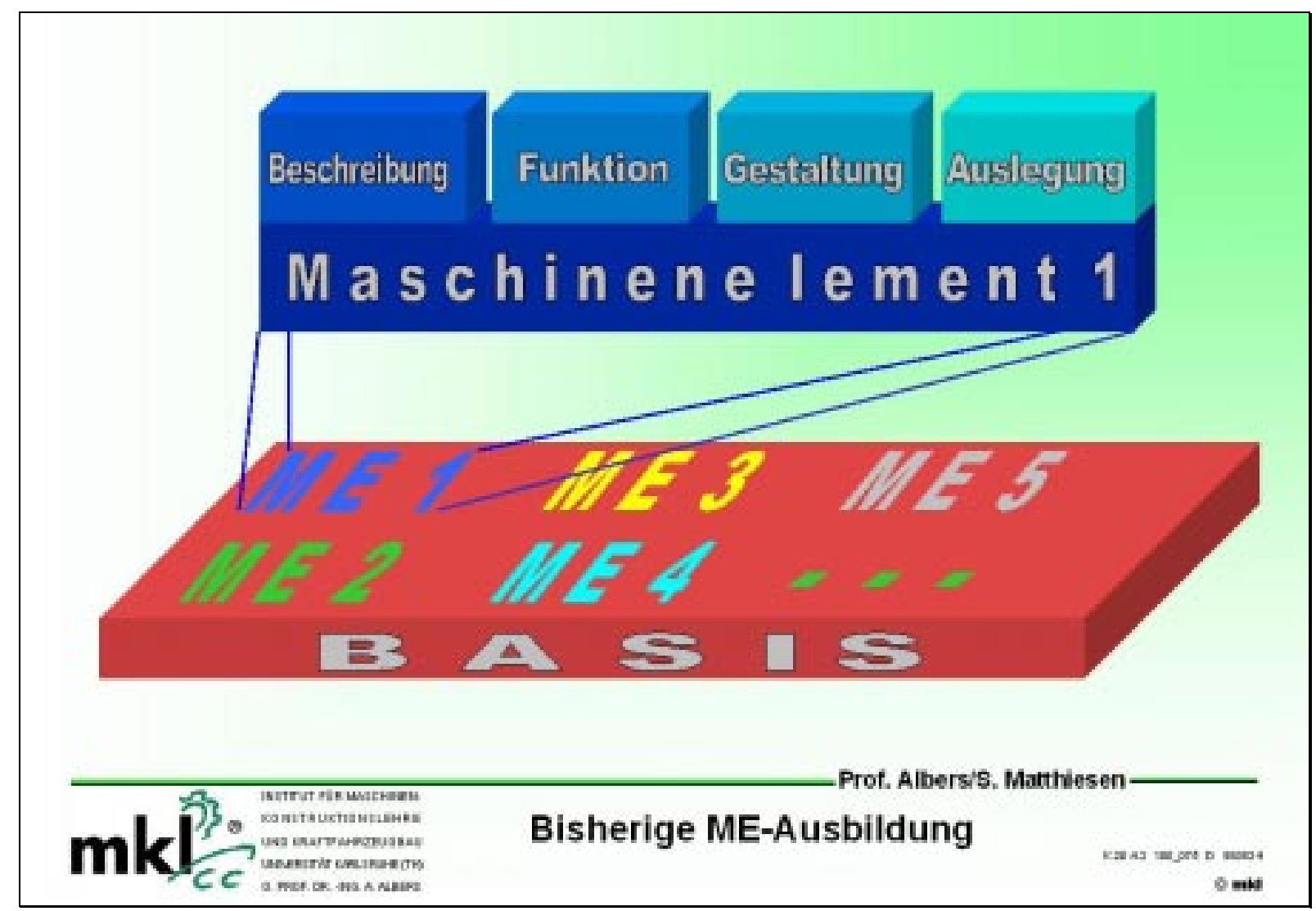

Abb.4: Struktur bisheriger Maschinenelementausbildungen

Durch die Vielzahl neuer Maschinenlemente ist eine vollständige Behandlung aller existierenden Maschinenelemente, bei gleichbleibender Vorlesungskapazität, nicht mehr möglich und auch nicht mehr sinnvoll. Das neue Karlsruher Vorlesungskonzept basiert auf einem völlig anderen Ansatz um den Studenten trotz stetig steigender Zahl von Maschinenelementen eine ganzheitliche und vollständige Maschinenelementelehre zu vermitteln:

Die Maschinenelemente werden von Anfang an auf einer höheren Abstraktionsebene aus konstruktionsmethodischer Sicht betrachtet. Sehr viele Maschinenelemente können bereits als System aus mehreren Komponenten aufgefaßt werden. Jedes 
Element eines Systems erfüllt dabei seine Funktion durch einen oder mehrere Kontakte zu einer anderen Systemkomponente. Die eigentliche Funktion und damit die gewünschte Wirkung wird also erst durch den Kontakt einer Fläche mit einer anderen Fläche realisiert. Diese Flächen werden somit zu Wirkflächen.

Jede Wirkfläche steht also zu irgendeinem Zeitpunkt mit einer anderen Wirkfläche in Kontakt um die Funktion des Maschinenelementes zu erfüllen. Die beiden Wirkflächen bilden ein Wirkfächenpaar. Beanspruchung, Auslegung und Gestaltung dieser Wirkflächen sind allein von der Funktion des Maschinenelementes, den Einsatzrandbedingungen und der mit dieser Wirkfläche in Kontakt kommenden anderen Wirkfläche abhängig. Wirkflächenpaare völlig unterschiedlicher Maschinenelemente werden häufig ähnlich ausgelegt, und gestaltet, da immer wieder gleiche Elementarfunktionen durch Wirkflächenpaare realisiert werden, wie zum Beispiel der geschmierte Kontakt unter hertzscher Pressung.

Die weitere Struktur des Maschinenelementes erfült lediglich die Funktion, die Wirkfläche in ihrer definierten Lage zu halten. Sie wird folgenden als Stützstruktur bezeichnet und muß nach ihrer Funktionserfüllung gestaltet und ausgelegt werden. Ähnlich der Wirkfläche ist also die Gestaltung und Auslegung der Stützstruktur allein von der Funktion und den Umgebungsbedingungen abhängig. 


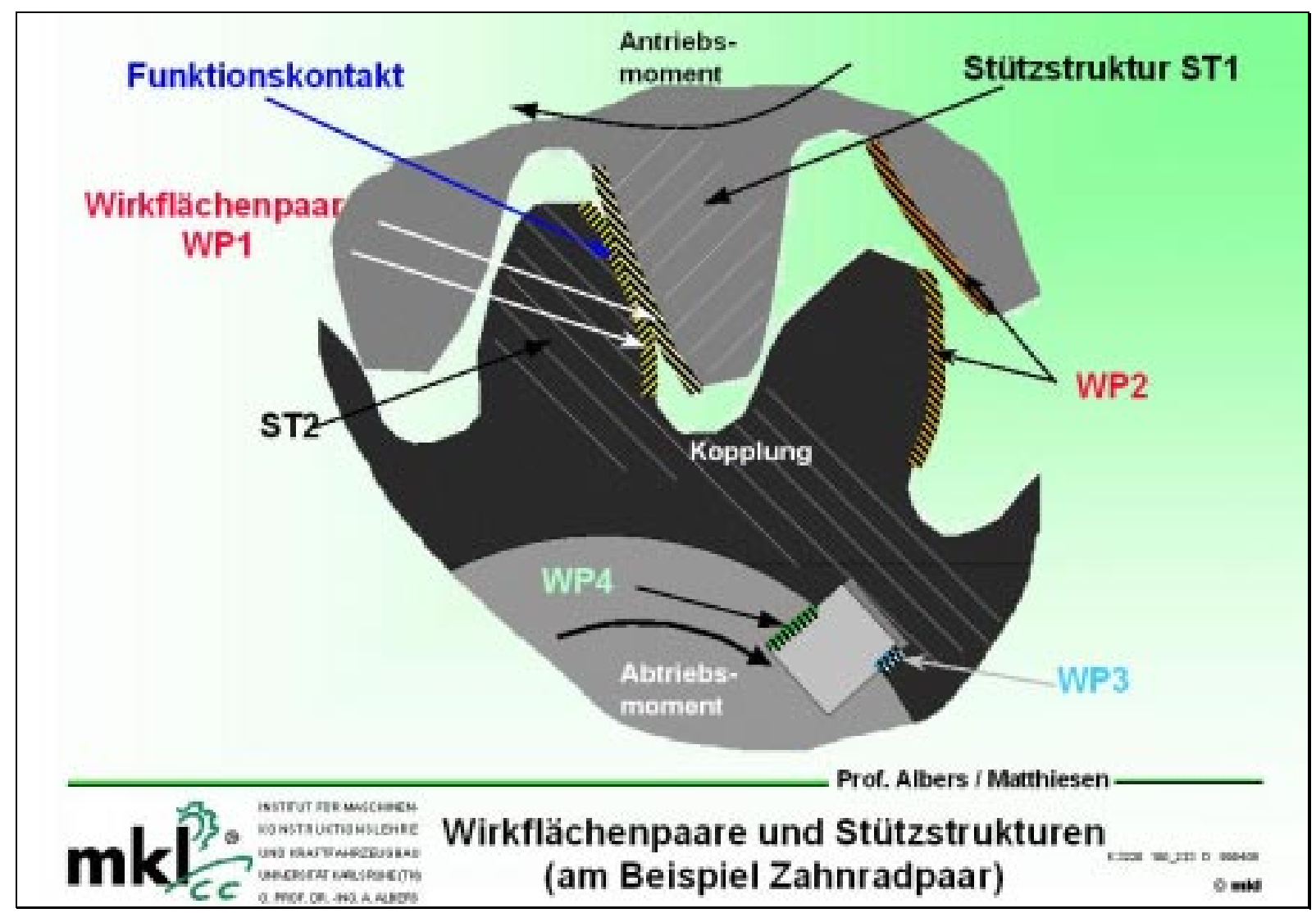

Abb.5: Theorie der Wirkflächenpaare und Stützstrukturen

Im neuen Vorlesungsansatz wird dem Studenten zu Beginn der Maschinenkonstruktionslehre die Theorie der Wirklächenpaare und Stützstrukturen vermittelt. Maschinenelmente werden also am Anfang der Veranstaltung auf eine hohe Abstraktionsebene gestellt. Gleichzeitig wir hierdurch die für den Maschinenbauingenieur so wichtige Fähigkeiten zur Abstraktion gelehrt und vertieft. Später wird die Theorie an ausgewählten Maschinenelementen exemplarisch und ausführlich belegt. Der Student lernt die abstrakte Theorie aus der Vorlesung auf ein konkretes Beispiel herunterzubrechen und anzuwenden. Die Maschinenelemente werden dabei zunächst immer aus der Sicht und dem Zusammenhang der Leitsysteme heraus betrachtet und damit sowohl in ihren Elementeigenschaften als auch in den Wechselwirkungen mit dem Gesamtsystem besprochen. Als Beispiel sei hier die Tellerfeder als Element der Anfahrkupplung genannt. Durch parallele Diskussion von Gestaltung-, Fertigungs-, Kosten- und Dimensionierungsgesichtspunkten wird eine ganzheitliche Sicht an Praxisbeispielen vermittelt. Der Schwerpunkt der Vorlesung unterscheidet sich damit stark vom Stoff 
klassischer Maschinenelementelehrbücher, die für das weitere Lernen von Faktenwissen im Selbststudium zur Verfügung stehen. Bei der Behandlung weiterer wichtiger Maschinenelemente kann sich der Dozent auf die exemplarisch erläuterten Maschinenelemente berufen und braucht diese Elemente nur kurz ansprechen, da der Student bereits gelernt hat, Wissen höheren Abstraktionsgrades auf konkrete Problemstellungen zu transformieren. Beispielsweise tritt zwischen der Systemkomponente Wälzkörper und der Systemkomponente Lageraußenring also im Wirkflächenpaar Wälzkörperoberfläche / Lagerlaufbahn Hertzsche Pressung auf. Im Wirkflächenpaar zweier miteinander kämmender Zahnräder, der Paarung zweier Zahnflanken, tritt ebenfalls Hertzsche Pressung auf. Folglich werden Zahnflanken und Wälzkörperoberflächen, auf einer gehobenen Abstraktionsstufe, auch ähnlich ausgelegt und gestaltet.

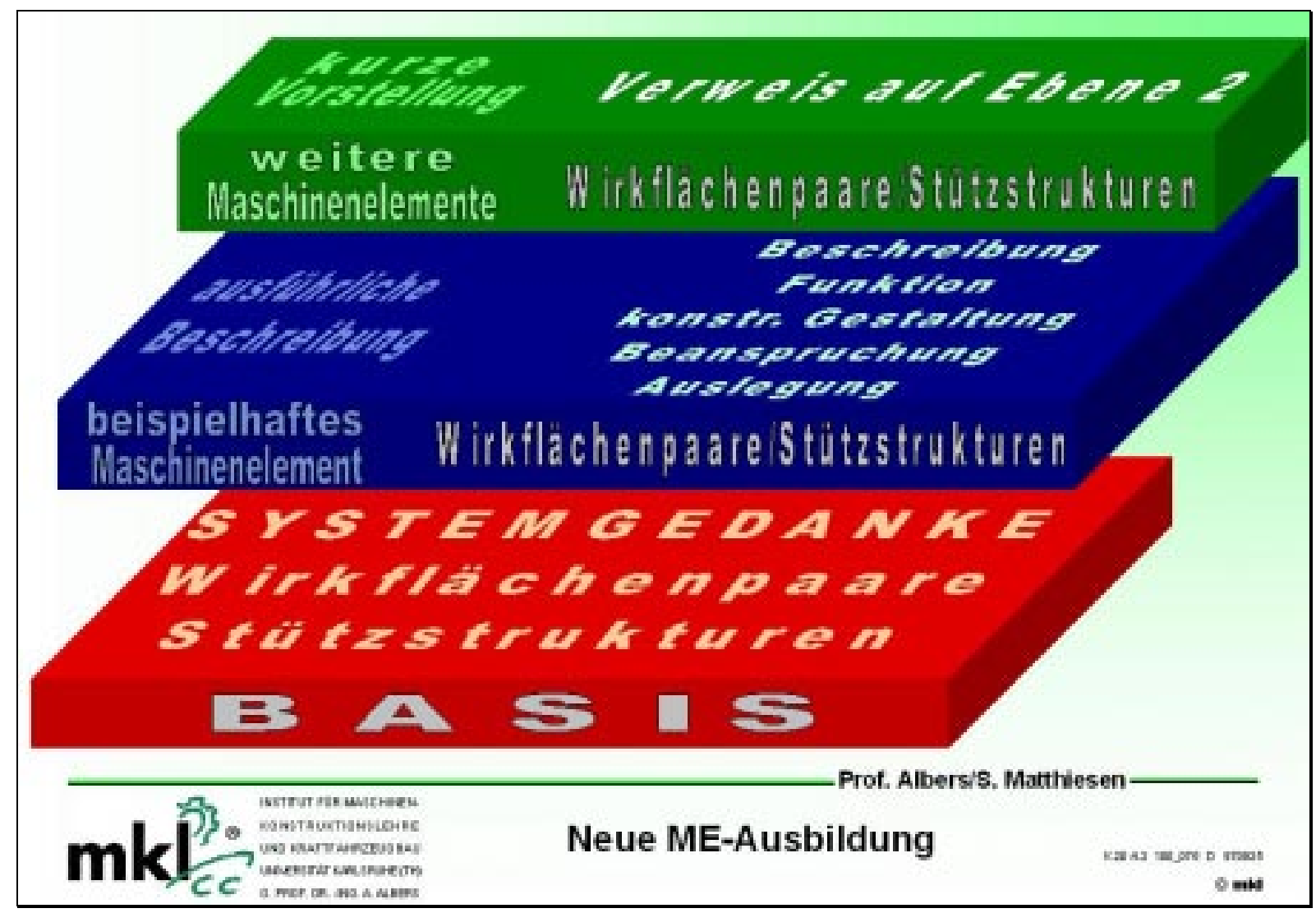

Abb.6: Das Ebenenmodell der neuen Maschinenkonstruktionsausbildung

Die Vorlesung hat nicht den Anspruch alle Maschinenelemente vollständig zu behandeln, sondern vermittelt vielmehr die Fähigkeit unbekannte Maschinenelemente und komplexe Maschinensysteme durch den Fokus auf 
Wirkflächenpaare und Stützstrukturen im Maschinensystem zu verstehen, zu analysieren und neue Elemente in bekanntes Grundlagenwissen einzuordnen. Damit wird die Fähigkeit zu selbständigen Synthese gefördert.

Rund $15 \%$ des Vorlesungsinhaltes behandelt nichtmechanische mechatronische Elemente und Systeme um so die Erweiterung der modernen Maschinenkonstruktion klar zu machen. Beispielsystem ist hier wieder der automatisierte Antriebsstrang.

Die Vorlesung wird vollständig unter Nutzung digitaler Präsentationstechnik durchgeführt. Damit wird es möglich auch digitale Videosequenzen in die Vorlesung einzubinden und unter extremer Zeitersparnis Wissen zu vermitteln.

\section{Übung}

Unter Übung wird nach dem neuen Lehrmodell generell eine Veranstaltung verstanden in der ein übungsleitender Assistent allen Studenten gleichzeitig im Frontalunterricht Wissen vermittelt. Der „Führungsstil“ des Lehrenden aus Sicht des Teammanagements ist dem der Vorlesung ähnlich dozentenorientiert und wenig interaktiv. Vereinfacht kann dieser Führungsstil auch als „autoritär und patriarchalisch" bezeichnet werden (Abb. 7). In der Übung sind Fragen und Anregungen von Seiten der Studenten zwar erwünscht, trotzdem kann aufgrund der großen Studentenzahlen (ca. 275 Studenten im Sommersemester '99) nur beschränkt darauf eingegangen werden.

In der Übung wird die Vorlesungstheorie aufgegriffen und vertieft. In Übungsaufgaben, die sich zum Großteil auf die Leitbauteile und Leitsysteme der Vorlesung und des Workshops beziehen, lernt der Student theoretisches Wissen auf konkrete Probleme anzuwenden und das verinnerlichte Wissen umzusetzen und zu transformieren. 


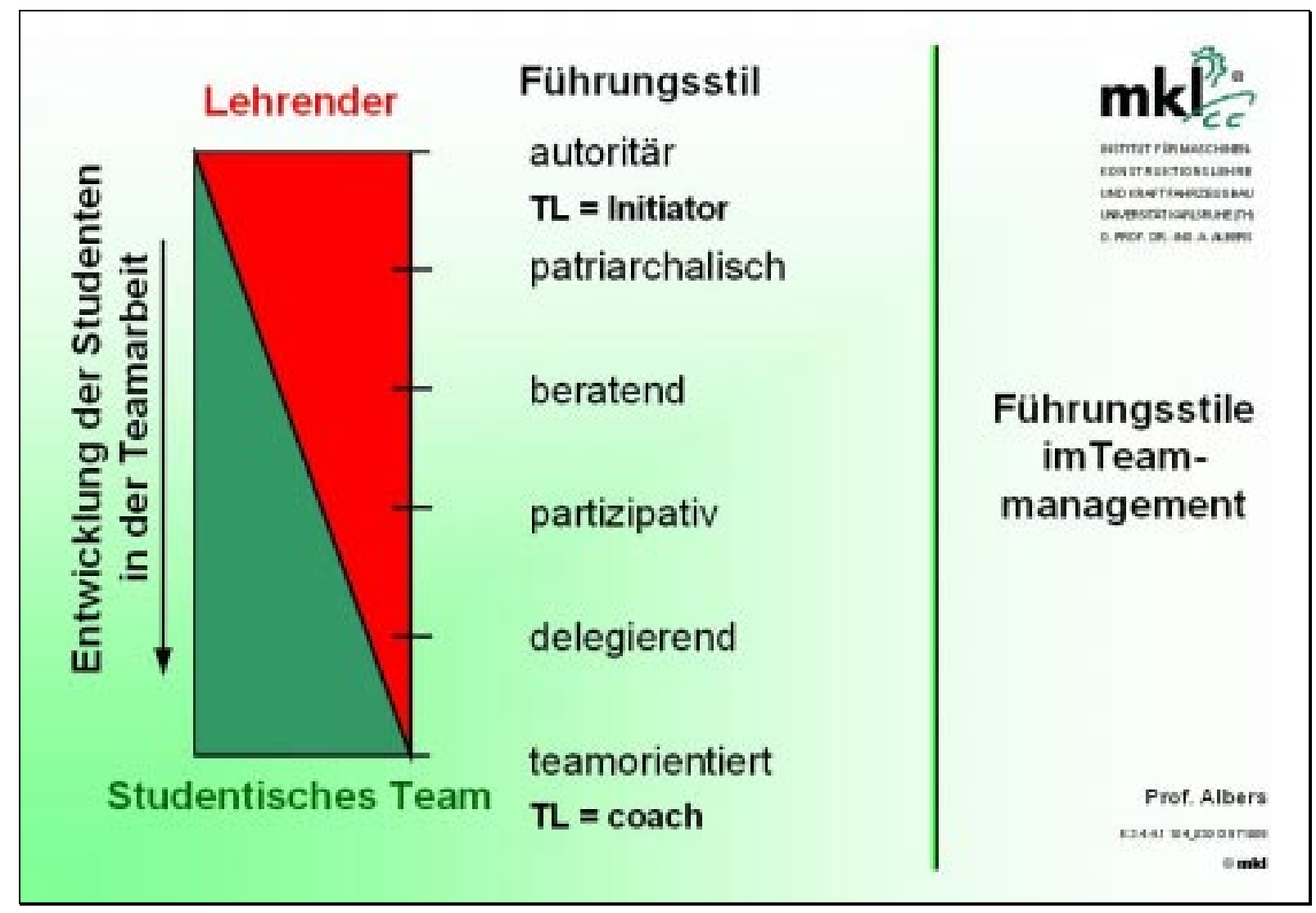

Abb.7: Führungsstile im Teammanagement in der Anwendung auf die Ausbildung

\section{Workshop}

Im Workshop sollen unter anderem die für den Ingenieur so wichtigen, oben bereits erwähnten, Softskills vermittelt werden. Es wird von Anfang an konsequent Teamarbeit verlangt. Konstruktion findet im Team unter selbständiger Aufgabenverteilung statt. Erfahrungen der einzelnen Teammitglieder müssen unter den anderen Teammitgliedern ausgetauscht werden. Die für die Studenten aus der Schule oft völlig ungewohnte Teamarbeit muß natürlich erst unter Anleitung geübt werden. Dazu stehen sechs Assistenten neben 18 studentischen Hilfswissenschaftlern während den wöchentlich stattfindenden Workshops bereit. Zu Beginn des Workshops greifen die Betreuer noch autoritär in die Teamarbeit der Studenten ein. Im zweiten und dritten Semester ziehen sich die Betreuer immer weiter aus der Problemlösung zurück und greifen nur noch beratend, partizipativ, bzw. delegierend in die Prozesse ein. Betreuung wird in den späteren Semestern also nur noch als Coaching verstanden. Dadurch wird eine ständig wachsende Selbständigkeit von Seiten der Studenten gefordert. 
In den ersten zwei Semestern werden die Studenten mit einem einfachen Leitsystem und den Leitbauteilen konfrontiert. Sie haben die Möglichkeit Getriebemotoren in 18 von Assistenten und Hilfswissenschaftlern begleiteten und 9 freien Workshops zu zerlegen und die verschiedenen Systemkomponenten zu analysieren.

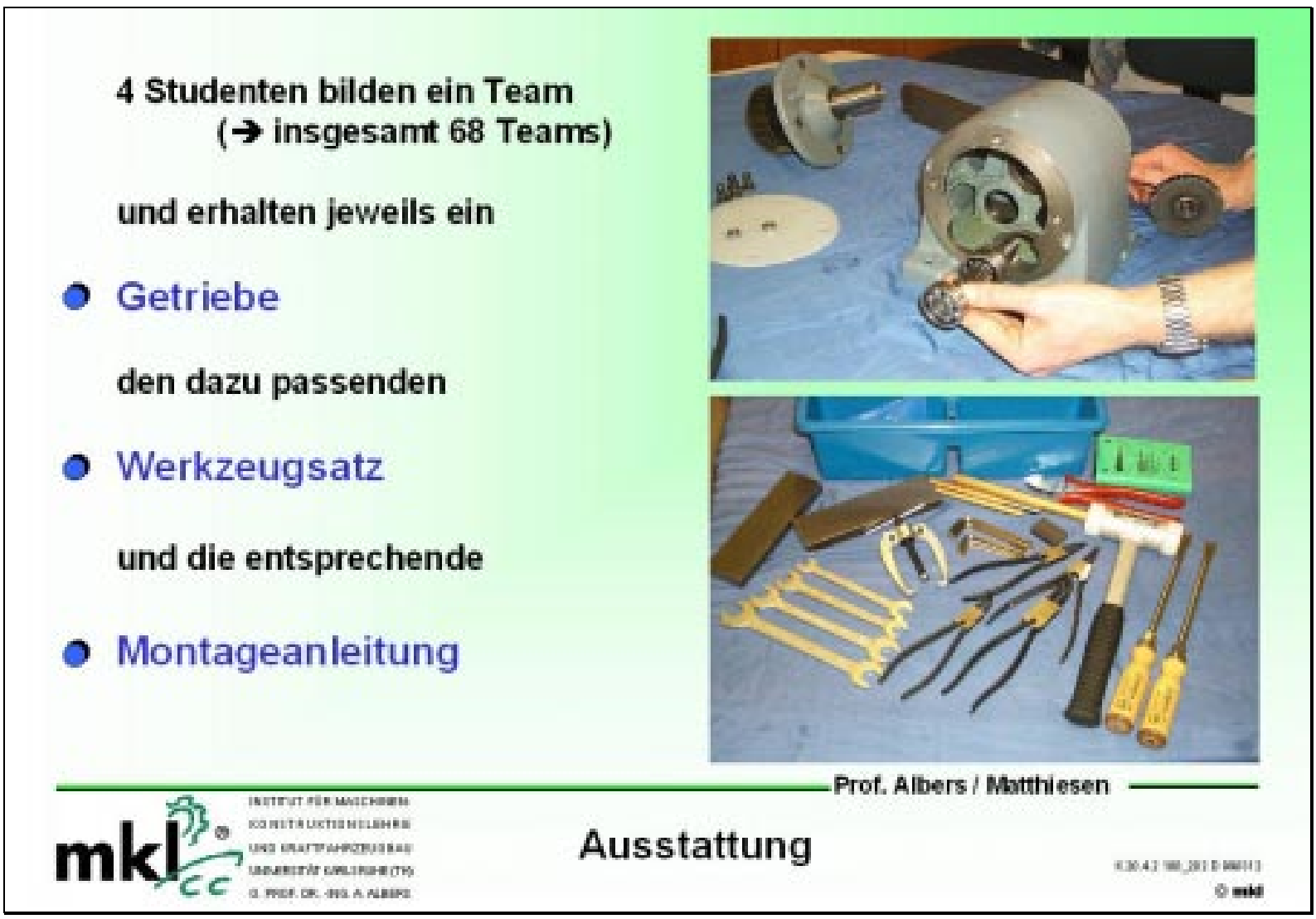

Abb.8: Leitbauteile im mkl-Workshop

Im ersten Semester werden im Workshop unter anderem Themen wie Technisches Freihandzeichnen, Analyse von Bauteilen in Gestalt und Funktion, Oberflächenanalysen und Messung unter Beachtung verschiedener Herstellprozesse, Passungsanalyse und erste Syntheseüberlegungen behandelt. 
3. Workshop

- Demontage der Welle 2

- Analyse der Lagerung von Welle 2 (Lagerart, Lagerfixierung, Kraftleitung)

- Vermessen der Welle 2

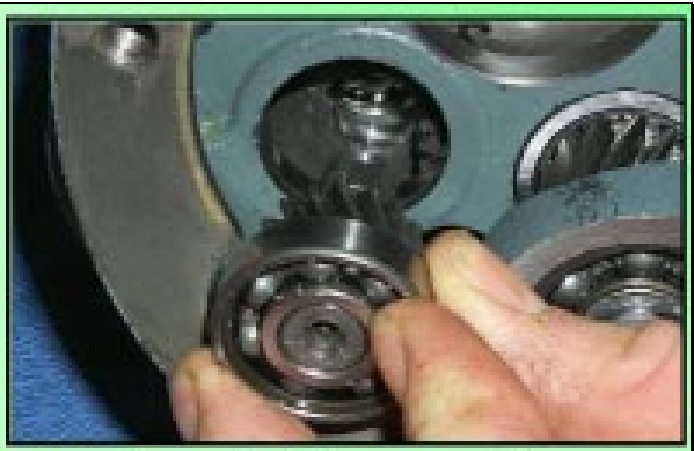

- Erstellung einer techn. Zeichnung (unter Anwendung der Klappregel)

- prüfgerechete Bemaßung der Zeichnung

- Kontrolle der Zeichnung durch Nachvollziehen eines Prüfvorganges

- Vergleich von 3D-Freihandskizze, Produktmodell und techn. Zeichnung

- Berechnung der Passung zwischen Lagerinnenring und Welle 2 (Umfangslast/Punktlast)

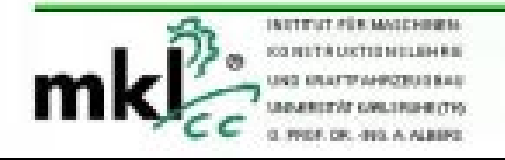

Workshopinhalte W3

Prot. Albers / Matthiesen

Abb.9: Beispiel für Workshopinhalte

Im zweiten und dritten Semester werden vor allem Maschinensysteme mit steigendem Komplexitätsgrad konstruiert und entworfen. Auch diese Arbeiten finden in studentischen Teams statt. Schnittstellen bei der Konstruktion legen die Teams selbst fest. Einzelkonstruktionen werden von den Studenten abgestimmt, zusammengeführt und dann von den Betreuern als Ganzes bewertet. Abschlußaufgabe ist eine Konstruktion aus dem industriellen Umfeld mit offener Problemstellung, deren Lösung auch den Betreuern, die nur noch delegierend in den Problemlösungsprozeß eingreifen, unbekannt ist.

\section{Das Darmstädter Lehrmodell}

Traditionell hat die Maschinenelemente-Ausbildung an der TU-Darmstadt (TUD) einen hohen Stellenwert. Zum Wintersemester 1998/99 wurde auf der Basis der Thesen und der Modelle des Arbeitskreises Maschinenelemente ein neues Lehr- und Lernmodell an der technischen Universität Darmstadt zur Vermittlung der Maschinenelemente entwickelt. 


\section{Das Konzept des Lehrmodells}

\section{Die Vermittlung des Systemgedankens}

Ein besonderes Anliegen war dabei die Vermittlung des Systemgedankens und die angemessene Berücksichtigung mechatronischer Maschinenelemente (Abb.10).

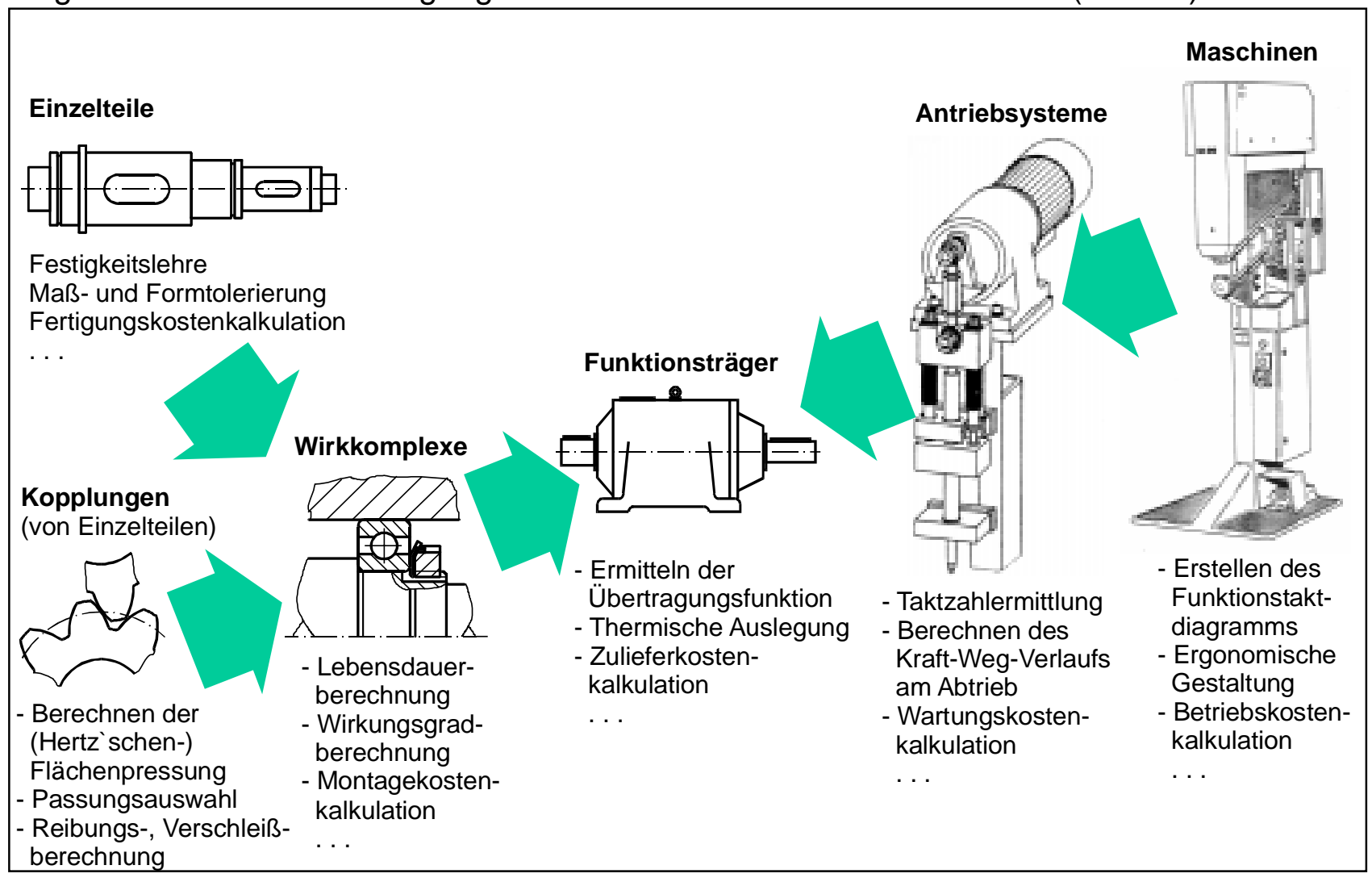

...

Abb.10: Problemorientierter Ansatz - Maschinenelemente und zugeordnete Methoden

Entgegen der üblichen Vorgehensweise soll die neue Ausbildung bei einem Überblick über technische Systeme beginnen und deren Komponenten in einem Systemzusammenhang darstellen. Erst wenn die Studierenden technische Systeme kennengelernt haben und die Vielfalt mechatronischer Komponenten ein- und zuordnen können (Top-Down-Ansatz) soll dann die vertiefte Behandlung der mechanischen Maschinenelemente vom Bauteil ausgehend bis zu den mechanischen Systemkomponenten (Bottom-Up-Ansatz) erfolgen. Diese Mischung aus Top-Down- und Bottom-Up-Ansatz soll einerseits die Voraussetzungen für das Auslegen und Gestalten mechanischer Maschinenelemente -z.B. Lastannahmennachvollziehbar machen. Andererseits soll damit das Verständnis bei den 
Studierenden geweckt werden, daß die Beherrschung der Auslegung und Gestaltung mechanischer Maschinenelemente eine Kernkompetenz eines jeden Maschinenbauingenieurs ist, die auch bei vielen nichtmechanischen Komponenten wie Elektromotoren, Hydraulikzylindern oder Pneumatikventilen unverzichtbar ist. Dieses Lehrkonzept wird auch dadurch erleichtert, daß sich viele Methoden zum Auslegen und Gestalten an Maschinenelementen bestimmter Komplexitätsstufen festmachen lassen (Abb.11).

\section{Die Integration nichtmechanischer (mechatronischer) Maschinenelemente}

Wie sind aber nun bei gleichem Stundenumfang die zusätzlichen Inhalte sinnvoll und verständnisfördernd unterzubringen? In der ganzen Breite und Tiefe können mechatronische Maschinenelemente keinesfalls vermittelt werden. Deswegen wurde an der TUD ein sogenanntes T-Modell der Lehrinhalte und Lernziele erarbeitet (Abb.12).

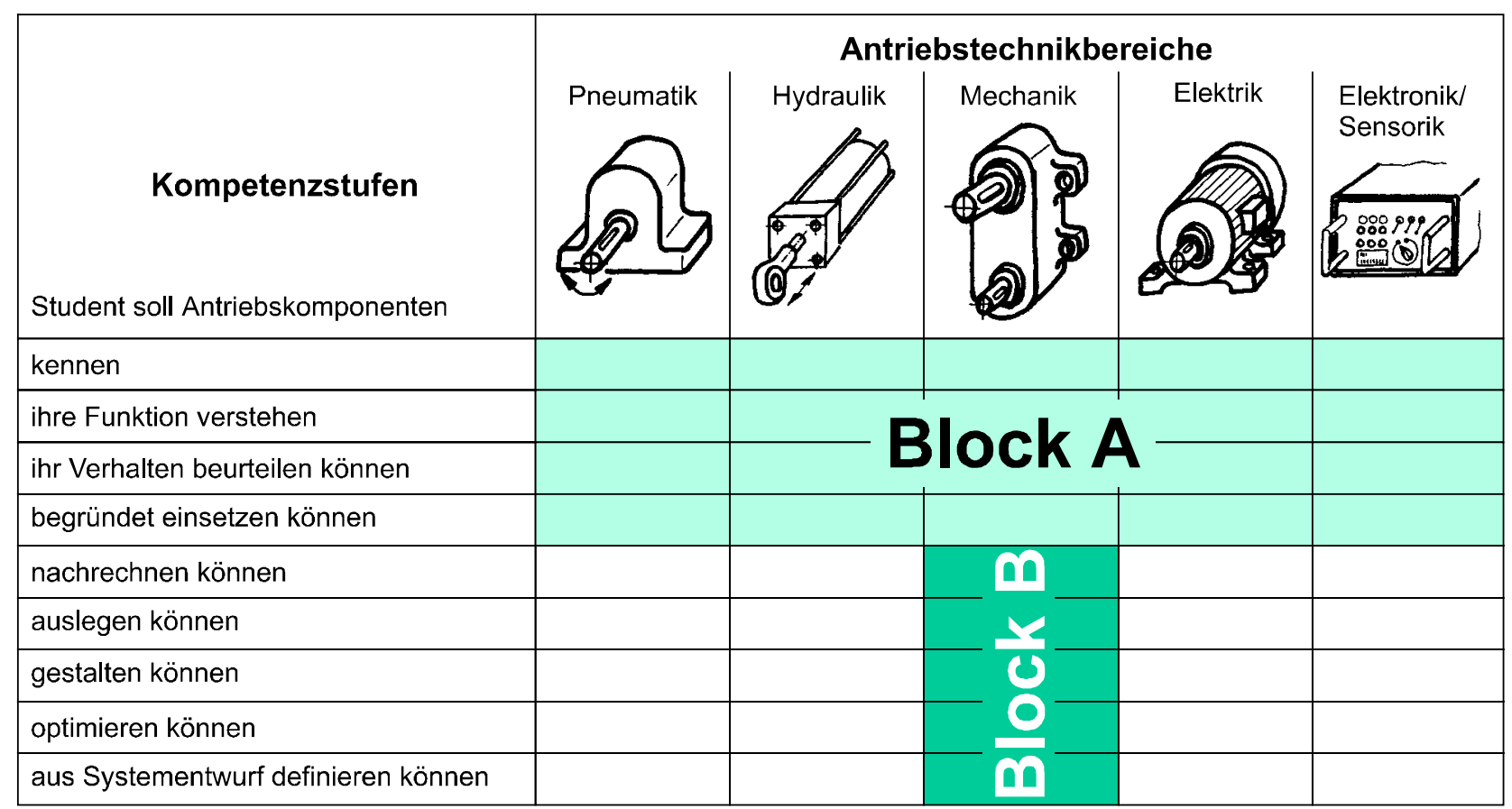

Abb.11: T-Modell der Lehrinhalte und Lernziele

Ein erster Block A der gesamten Maschinenelemente-Ausbildung soll mechatronische Elemente in voller Breite, allerdings nur in reduzierter Tiefe 
vermitteln. Daran soll sich dann der Block B anschließen, in dem die Studierenden an mechanischen Maschinenlementen ihre Kompetenzen vergleichbar zur bisherigen Veranstaltung vertiefen können.

\section{Der Systementwurf in Projektarbeit}

Neben dem Systembezug und der angemessenen Berücksichtigung nichtmechanischer Maschinenelemente bereits im Grundstudium ist an der TUD ein drittes, didaktisches Element aufgegriffen worden, das im ersten Lichtenthaler Workshop diskutiert wurde. Dort zeigte sich, daß durchaus unterschiedliche Lehrund Lernmodelle je nach konkreter Situation an den einzelnen Hochschulen sinnvoll sein können. Besonders faszinierende Perspektiven gerade für eine Maschinenelemente-Ausbildung bietet die Projektarbeit, in dem die Studierenden in Teams eine komplexere Aufgabenstellung ganzheitlich bearbeiten. Sie sollen dabei das bisher erworbene Fach- und Methodenwissen einbringen, die Wechselwirkungen zwischen Gestaltung und Berechnung, zwischen Detail und Ganzem kennenlernen und erstmals im Grundstudium die Synthese eines komplexeren, technischen Systems durchführen.

Diese Lernziele werden von den Verantwortlichen für so wesentlich erachtet, daß der dritte Block der neuen Veranstaltung komplett für die Projektarbeit zur Verfügung stehen soll. Nach einer einführenden Vorlesung zur Planung, Durchführung und Steuerung einer Projektarbeit sollen die Studierenden ein mechatronisches System entwickeln, es hinsichtlich Dynamik und Festigkeit auslegen, technisch und wirtschaftlich optimal gestalten und zumindest wesentliche Komponenten davon in 3D-CAD modellieren. Die Projektarbeit wird begleitet von Zwischen- und Endpräsentationen, beide Professoren und alle wissenschaftlichen Mitarbeiter und Tutoren betreuen sie. Innerhalb des Blocks C sollen keine Vorlesungen stattinden, die Vorlesungszeit wird für die Betreuung der Projektgruppen genutzt.

\section{Durchführung}

Abb. 12 zeigt den Ablauf der neuen Maschinenelemente-Veranstaltung, wie sie seit dem WS 98/99 an der TUD durchgeführt wird. Block A mit 40\% des gesamten Stundenumfangs wurde Mitte Januar abgeschlossen. Zur Zeit (April 99) wird Block B mit ebenfalls 40\% Zeitanteil durchgeführt während der Block C mit 20\% Zeitanteil Anfang Juni 99 beginnt und derzeit noch in Vorbereitung ist. 


\begin{tabular}{|c|c|c|}
\hline 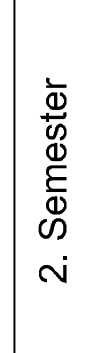 & $\begin{array}{l}\text { Einführung } \\
\text { in das } \\
\text { rechner- } \\
\text { gestützte } \\
\text { Konstruieren } \\
\text { (CAD) } \\
\text { 1V / 3Ü }\end{array}$ & $\begin{array}{l}\text { Lernziele: } \\
\text { - Kompetenz im 3D-Modellieren } \\
\text { - Perspektiven einer 3D-Modellierung } \\
\text { - Skizzierfähigkeit } \\
\text { - Räumliches Vorstellungsvermögen } \\
\text { - Zweckmäßiges Skizzieren und Modellieren } \\
\text { - Zeichnungsregeln } \\
\text { - Prozeßkettendenken }\end{array}$ \\
\hline
\end{tabular}

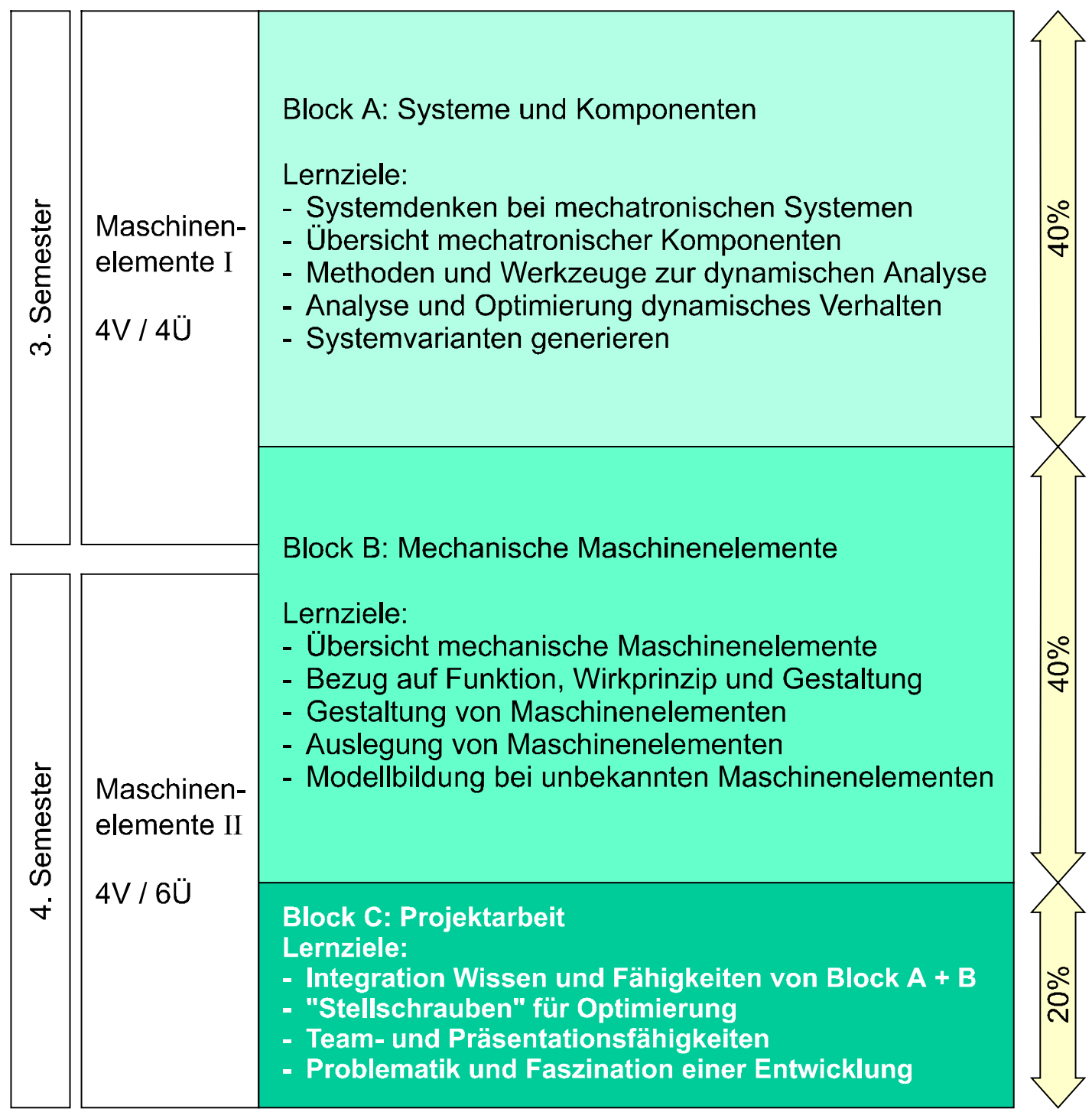

Abb.12: Grobgliederung der neuen Maschinenelemente-Ausbildung

Während der Block B auf den bisherigen, allerdings wesentlich konzentrierter gefaßten Inhalten beruht wurde der Block A völlig neu konzipiert (Tabelle 1). 


\begin{tabular}{|c|c|c|}
\hline Wintersemester & & \\
\hline \begin{tabular}{|l|l|} 
DSt. & Vorlesung \\
\end{tabular} & Übung & Mod \\
\hline
\end{tabular}

\begin{tabular}{|c|c|c|}
\hline \multicolumn{3}{|c|}{ Block A: Mechatronische Systeme und Komponenten } \\
\hline 1. & 1. Einführung in die neue ME-Lehre & \multirow{7}{*}{$\begin{array}{l}\text { Block I } \\
\text { Organisation } \\
\text { Blockschaltbilder } \\
\text { Differentialgleichungen } \\
\text { Numerisches integrieren } \\
\text { Einstieg in Matrixx }\end{array}$} \\
\hline 2. & 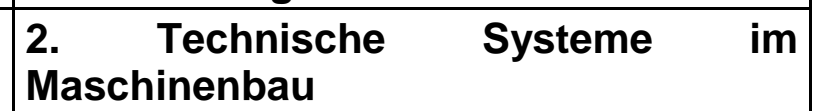 & \\
\hline 3. & Systembeschreibung, & \\
\hline 4. & \multirow{3}{*}{$\begin{array}{l}\text { 3. Grundlagen für Technische Systeme } \\
\text { Funktion und Verhalten } \\
\text { Mechatronische Modelle }\end{array}$} & \\
\hline 5. & & \\
\hline 6. & & \\
\hline 7. & \multirow{3}{*}{$\begin{array}{l}\text { Dynamik mechatronischer Systeme, } \\
\text { Regelkreis } \\
\text { Simulation, Werkzeuge } \\
\text { Grundlagen der Festigkeitslehre }\end{array}$} & \\
\hline 8. & & \multirow{8}{*}{$\begin{array}{l}\text { Block II } \\
\text { Modelle von } \\
\text {-mechanischen } \\
\text { Komponenten } \\
\text {-Aktoren und Sensoren } \\
\text { mathematische Herleitung } \\
\text { und Umsetzung am } \\
\text { Rechner } \\
\text { Simulation }\end{array}$} \\
\hline 9. & & \\
\hline 10. & $\begin{array}{l}\text { 4. Prozesse und Prozeßkomponenten } \\
\text { (Abtrieb) }\end{array}$ & \\
\hline 11. & 5. Energiespeicher & \\
\hline 12. & 6. Aktoren Grundlagen, Beispiele & \\
\hline 13. & 6.1 Elektromagnetische Aktoren & \\
\hline 14. & 6.2 Elektrodynamische Aktoren & \\
\hline 15. & $\begin{array}{|lll|}6.3 & \text { Pneumatische } \\
\text { Aktoren }\end{array}$ & \\
\hline 16. & 6.4 Mechanische Aktoren (Getriebe) & arametervariation \\
\hline 17. & \begin{tabular}{|l|l}
6.5 & Stellglieder \\
Trennkupplungen)
\end{tabular} & \\
\hline 18. & 7. Sensoren Grundlagen Beispiele & \\
\hline 19. & $\begin{array}{l}\text { Sensoren für } \\
\text { Bewegungsgrößen }\end{array}$ & Block III \\
\hline 20. & Sensoren für andere Größen & Mechatronisches \\
\hline 21. & $\begin{array}{l}\text { 8. Regler und Steuerungen Grundlagen, } \\
\text { Beispiele }\end{array}$ & Gesamtsystem analysieren \\
\hline 22. & $\begin{array}{l}\text { Analoge, digitale Regler, A/D- und. D/A- } \\
\text { Wandler }\end{array}$ & \\
\hline
\end{tabular}

Tabelle 1: Gliederung der Vorlesungen und Übungen zu Block $A$

Von den zur Verfügung stehenden 22 Doppelstunden wurden 3 für einen Überblick, 6 für die Vermittlung der notwendigen Grundlagen (z.B. Dynamik, Regelungstechnik, Simulation) genutzt und in den restlichen 13 Doppelstunden die einzelnen Systemkomponenten behandelt. Jedes System und jede Komponente wurden dabei 
immer wieder in einen Systemzusammenhang gestellt, wie er in Abb.13 4 dargestellt ist.

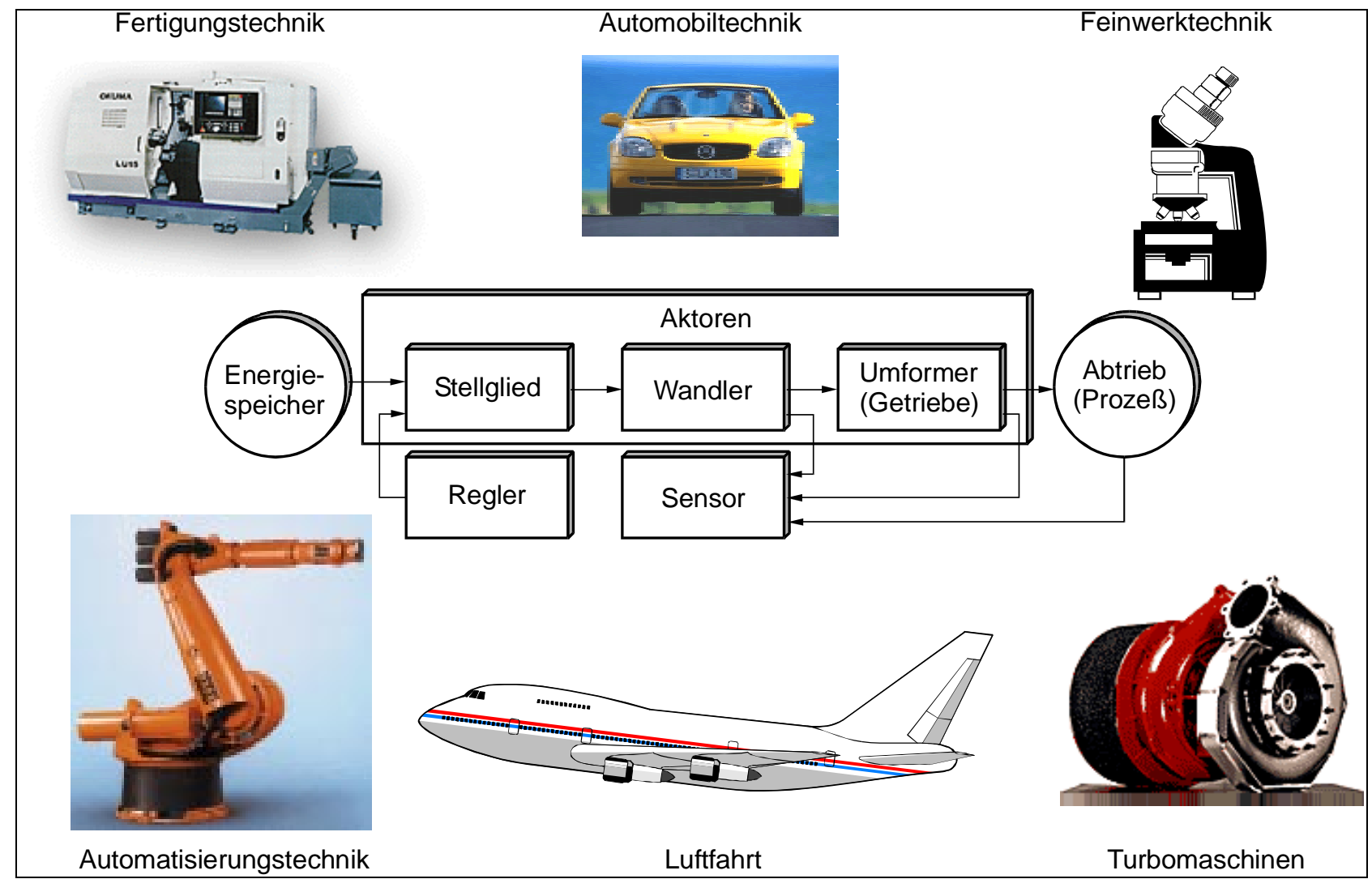

Abb.13: Technische Systeme und Komponenten

In den Übungen zu Block A erwarben die Studierenden zuerst die Kompetenz, mit MatrixX einfache Simulationen durchzuführen und erweiterten dann schrittweise ihre Fähigkeiten von der dynamischen Analyse bekannter Systeme auf deren Optimierung. Leitbeispiel war dabei eine elektromechanische Kompensationswaage, die sowohl als Produkt als auch als vereinfachtes Funktionsmuster für den Vergleich Simulation - Experiment zur Verfügung gestellt wurde.

\section{Erste Ergebnisse und Erkenntnisse}

Die gesamte Veranstaltung wird pädagogisch/didaktisch von der Hochschuldidaktischen Arbeitsstelle (HdA) der TUD betreut. Im Rahmen der Betreuung findet nach jeder Vorlesung, an der beide Hochschullehrer und die wissenschaftlichen Mitarbeiter teilnehmen sofort eine "Manöverkritik" statt. Zusätzlich 
werden Studenten regelmäßig interviewt, am Ende eines jeden Blocks findet eine detaillierte Befragung aller Studierenden mit Fragebögen statt.

Die neue Konzeption der Maschinenelemente-Veranstaltung findet bei den Studierenden durchweg positive Resonanz. Sie wird als notwendig und hilfreich für das gesamte Studium und als sinnvoll für den späteren Ingenieurberuf eingeschätzt. Trotz des einhellig als hoch eingeschätzten Schwierigkeitsgrades und des mit dieser, wie wohl mit jeder anderen Maschinenelemente-Veranstaltung verbundenen, großen Zeitbedarfs werden unvermeidbare Mängel dieses ersten Durchlaufs von den Studierenden erstaunlich "kulant" behandelt. Hervorzuheben ist auch als erstes Zwischenergebnis, daß die neue Veranstaltung insbesondere bei den leistungsstärkeren Studierenden und denjenigen mit abgeschlossener Lehre besonders positiv abschneidet.

\section{Zusammenfassung}

Im Arbeitskreis Maschinenelemente der WGMK wurden wichtige Thesen für die Weiterentwicklung der Maschinenkonstruktionslehre erarbeitet und die notwendigen Schritte zur Anpassung der Maschinenkonstruktionslehre an die heutigen wissenschaftlichen und industriellen Erfordernisse formuliert.

Die Thesen bilden eine gemeinsame Basis auf der Lehr- und Lernmodelle aufgebaut werden können. Diese müssen die individuellen Situationen der jeweiligen Universitäten und die Erfahrungen sowie Forschungsschwerpunkte des verantwortlichen Hochschullehrers berücksichtigen. An den Universitäten Darmstadt (TUD) und Karlsruhe (TH) werden die neuen Lehr- und Lernmodelle bereits umgesetzt. Die Universität des Saarlandes /6/ wird im Wintersemester 99 damit beginnen. Positive Stellungnahmen zu den neuen Lehrkonzepten sowohl aus Studenten- als auch aus Industriekreisen bestätigen die neuen Ansätze und bestärken uns, den eingeschlagenen Weg zur Weiterentwicklung der Maschinenkonstruktionslehre konsequent und zielorientiert auf Grundlage der Thesen der WGMK weiterzugehen. Wir sind der festen Überzeugung, daß das Engagement in der Lehre ganz im Sinne unseres verehrten, so früh verstorbenen Kollegen Beitz ist. 


\section{Literatur}

/1/ Albers, A..: Wohin steuert die Maschinenkonstruktionslehre ?,Jahrestagung der WGMK 1996. Universität Karlsruhe (TH) 1996.

/2/ Albers, A.; Birkhofer, H.; Die Zukunft der Maschinenelementlehre; Tagungsunterlagen zum Workshop Heiligenberg; 23.04 u. 24.04.97; Universität Karlsruhe u. Universität Darmstadt; 1997.

/3/ Albers, A.; Birkhofer, H.; Neue Lehre; Tagungsunterlagen zum 1. Workshop Lichtental; 09.02 u. 10.02.98; Universität Karlsruhe u. Universität Darmstadt; 1998.

/4/ Albers, A.; Birkhofer, H.; Neue Lehre; Tagungsunterlagen zum 2. Workshop Lichtental; 24.02 u. 25.02.99; Universität Karlsruhe u. Universität Darmstadt; 1999.

/5/ Albers, A.; Simultaneous Engineering an einem Beispiel aus der Kraftfahrzeugzulieferindustrie; EK- VIP Führungskräftetreffen des VDI am 18. Juni 1993 in München, Tagungsband, VDI Verlag.

16/ Weber,C.: Activities in a New Way of Teaching Machine Elements in Germany. International Design Conference - Design'98, Dubrovnik, May 19-22, 1998 